\title{
CLIMA ORGANIZACIONAL EN UNA EMPRESA CERVECERA: UN ESTUDIO EXPLORATORIO
}

\author{
ORGANIZATIONAL CLIMATE IN A BEER COMPANY: AN EXPLORATORY STUDY \\ Beatriz Acosta U. ${ }^{1}$ y Cyntia Venegas G. \\ Universidad del Papaloapan, Campus Tuxtepec, México \\ (RECIBIDO El 18/01/2010, ACEPTAdo el 08/06/2010)
}

\begin{abstract}
RESUMEN
Los resultados que se presentan forman parte de la tesis de maestría de Cyntia Venegas Gómez (2010). El objetivo fue identificar el clima organizacional en una empresa cervecera, a partir del cuestionario de clima organizacional de Litwin y Stringer (1968).

Participaron 49 trabajadores de la empresa a quienes se les administró el Cuestionario antes mencionado, el cual consta de 53 reactivos distribuidos en 9 escalas.

Los trabajadores percibieron un buen clima en apoyo, estructura, riesgo, responsabilidad y calor y tuvieron una percepción negativa en conflicto, identidad, estándares de desempeño y recompensa.
\end{abstract}

Se encontraron diferencias estadísticas con respecto al sexo en recompensa y estándares de desempeño y no se encontraron diferencias en cuanto a la edad de los trabajadores.

Palabras clave: Clima organizacional, cuestionario de Litwin y Stringer, diferencias en cuanto al sexo y la edad.

\begin{abstract}
Results presented are part of Cyntia Venegas Gómez Master thesis (2010). Aim was to identify organizational climate in a beer company from Litwin and Stringer's organizational climate questionnaire, 1968.

Participated 49 workers which answered Litwin and Stringer Questionnaire. This has 53 items, divided in 9 scales.

Workers perceived a good climate in support, structure, risk, responsibility and warmth and bad climate in conflict, identity, standards and reward.

Statistical differences were founded regarding to sex in reward and standards and there weren't statistical differences in relation to age of the workers.
\end{abstract}

Key words: Organizational climate, Litwin and Stringer's questionnaire, differences regarding to sex and age.

1 Profesora-Investigadora Titular "A" Universidad del Papaloapan, Campus Tuxtepec, Oaxaca, México.

E-mail: bacosta@unpa.edu.mx 


\section{INTRODUCCIÓN}

Un empleado no opera en el vacío, lleva consigo al trabajo ciertas ideas preconcebidas sobre sí mismo, quién es, qué merece y qué es capaz de realizar. Estos preconceptos reaccionan con diversos factores relacionados con el trabajo, tales como el estilo de su jefe, la rigidez de la estructura organizacional y la opinión de su grupo de trabajo para determinar cómo ve su empleo y su ambiente. En otras palabras, el desempeño del empleado se gobierna no solo por su análisis objetivo de la situación, sino también por sus impresiones subjetivas del clima en que trabaja (Dessler, 1998).

En este sentido, los empleados establecen intercambios con su medio ambiente y mantienen un equilibrio dinámico con éste. Estos tienen necesidad de información proveniente de su medio de trabajo, a fin de conocer los comportamientos que requiere la organización y alcanzar así un nivel de equilibrio aceptable con el mundo que le rodea (Brunet, 1992).

De ahí que las organizaciones son entornos psicológicamente significativos, en donde se ven implicados la consideración de los trabajadores, de los grupos dentro de la organización y de otras formaciones sociales, los cuales son considerados como ambientes del de los propios trabajadores y estos están sujetos a la conducta organizacional (Peiró, 1985).

El empleado aparece entonces como inmerso dentro de un clima determinado por la naturaleza particular de la organización. Así la predicción del comportamiento individual basado estrictamente en las características personales es insuficiente y lleva muchas veces a concluir que el comportamiento depende en parte de la situación.

El constructo de clima organizacional conocido también como "atmósfera", "ambiente" o "aire" (Olaz, 2009) fue introducido por primera vez en la psicología organizacional por Gellerman en 1960 (citado en Brunet, 1992). No obstante, se le confunde con otros conceptos como: cultura, satisfacción o calidad de vida (Gómez, 2004).

Los estudios realizados en torno al tema en cuestión constatan la importancia que el clima ha alcanzado en el contexto de las organizaciones y, a pesar de ello, no existe un acuerdo generalizado sobre el significado y alcance del término (Olaz, 2009; García y Sánchez, 2008; Gómez, 2004, Jaime y Araujo, 2009).

La formación del clima es uno de los aspectos más importantes en la comprensión de este concepto. Los enfoques planteados para explicar este aspecto del clima se estructuran a partir de la taxonomía utilizada para clasificar las definiciones sobre el clima (Moran y Volkwein, 1992 en Jaime y Araujo, 2009, García y Sánchez, 2008; DeStefano, Clark, Gavin y Potter, 2005).

Enfoque estructural. El clima organizacional se describe como el conjunto de características permanentes que definen a una organización, la distinguen de otra, e influyen en el comportamiento de las personas que la conforman.

Enfoque subjetivo o perceptual. El clima es un proceso psicológico que describe las condiciones de la organización. Son las características de los individuos las que determinan las percepciones del clima.

Enfoque interactivo. Se refiere a la interacción de los individuos como respuesta dada a las situaciones objetivas y compartidas de la organización. 
Enfoque cultural. El clima se crea por un grupo de interacciones individuales que comparten un marco común de referencia, como es la cultura organizacional. La formación del clima está condicionada por los principios y valores que impregna la organización.

Dentro del clima organizacional sobresalen dos aspectos: la multidimensionalidad y la sectorialidad (Sánchez y García, 2004). La primera alude al hecho de que, aún formando una estrecha unidad, el clima laboral se compone de múltiples aspectos, siendo incapaz ninguno de ellos de explicar o absorber la totalidad del concepto, de sus efectos y consecuencias.

La sectorialidad matiza el concepto de globalidad que tiene el clima laboral. Si bien el clima es una variable que distingue a las organizaciones como un todo entre sí, el clima no es homogéneo en toda la organización, es decir, existen "microclimas organizacionales" que responden a circunstancias, situaciones y personalidad peculiares y que hacen que departamentos de una misma organización puedan tener climas muy diferentes entre ellos.

El clima equivale entonces a la medida de los climas reunidos en todos los departamentos y es la resultante de todos los microclimas que lo componen. Los determinantes físicos como la estructura, el tamaño, la tecnología son aplicables a todas las unidades y las dimensiones; sin embargo, estos se perciben comparativamente por los empleados (Helriegel, Slocum y Woodman,1999 en Gómez, 2004).

A decir de las dimensiones propuestas para la medición del clima laboral, destacan por sus contribuciones teóricas, las aportaciones de Halpin y Crofts (1963), Forehand y Gilmer (1964), Likert (1967), Meyer (1968), Schneider y Bartlett (1968), Friedlander y Margulies (1969), Lawler, Payne, Pheysey y Pugh (1971), Pritchart y Karasick (1973), Hall y Oldham (1974), Gavin (1975) y Steers (1977) citados en Brunet (1992).

El número de dimensiones que se encuentran es bastante heterogéneo; autores como Payne, Pheysey y Pugh (1971) proponen dos aspectos determinantes del Clima Organizacional; no obstante, Pritchard y Kurasich (1973) proponen una lista de once dimensiones (Brunet, 1992).

A pesar de dicho desacuerdo respecto de las diferentes dimensiones del clima, en la mayor parte de los cuestionarios utilizados se distinguen por ciertas dimensiones comunes entre las que destacan: a) nivel de autonomía individual que viven los actores dentro del sistema, b) el grado de estructura y de obligaciones impuestas a los miembros de una organización por su puesto, c) el tipo de recompensa o de remuneración que la organización otorga a sus empleados, d) la consideración, el agradecimiento y el apoyo que un empleado recibe de sus superiores (Rodríguez, 1999 en Gómez, 2004).

Se han desarrollado diversos cuestionarios que intentan medir el clima organizacional. Según Toro (2001) dentro de las encuestas más importantes reportadas en la literatura técnica se encuentran: a) inventario de clima psicológico de Gavin y Howe, b) índice de clima organizacional de Stern, c) cuestionario de clima organizacional de James y Sells, d) encuesta de calidad de empleo de Kahn, e) cuestionario de Michigan de evaluación organizacional de Camman, f) escala de ambientes de trabajo de Moos, g) cuestionario descriptivo del clima organizacional de Halpin y Crofts, h) escala del ambiente universitario 
de Pace, i) cuestionario de salud organizacional de Milles y j) cuestionario descriptivo del perfil del clima organizacional de Kettering.

De estos cuestionarios se derivan las siguientes categorías: relaciones sociales y de autoridad, tarea, claridad, retribución, retos, riesgos y participación.

Brunet (1992) menciona otra serie de cuestionarios, los cuales son: el cuestionario de Likert, el cuestionario de percepción del clima al interior de las compañías de seguros de Schneider y Bartlett, el cuestionario de Pritchard y Karasick, el cuestionario organizacional para el dominio escolar de Halpin y Crofts, el cuestionario llamado Survey of organizations de Bowers y Taylor, el Cuestionario adaptado a las empresas escolares de Crane y el Cuestionario de clima organizacional de Litwin y Stringer.

Dentro de las escalas de estos instrumentos se encuentran: métodos de mando, motivación, comunicación, influencia, toma de decisiones, planificación, control, rendimiento y perfeccionamiento, desempeño, apoyo, estructura, implicación, relaciones sociales, cohesión, conflictos, cooperación, autonomía, satisfacción, estatus, flexibilidad, innovación, obstáculos, intimidad, espíritu, importancia en la producción, tarea, confianza, consideración, apertura a los cambios tecnológicos, presión, confort, misión, responsabilidad, recompensa, riesgo e identidad.

\section{Teoría del clima organizacional de Litwin y Stringer}

Litwin y Stringer (1968) fueron los primeros autores en conceptualizar el clima organizativo bajo la perspectiva perceptual. Para ellos, el clima es un conjunto de propiedades del entorno de trabajo que son susceptibles de ser medidas percibidas directa o indirectamente por los trabajadores que vive y trabaja en dicho entorno y que influye en su comportamiento y motivación.

La teoría de Litwin y Stringer (1968) intenta explicar importantes aspectos de la conducta de los individuos que trabajan en una organización utilizando los conceptos como motivación y clima. Los autores tratan de describir los determinantes situacionales y ambientales que más influyen sobre la conducta y percepción del individuo.

Litwin y Stringer postulan la existencia de nueve dimensiones que explicarían el clima existente en una determinada empresa. Cada una de estas dimensiones se relaciona con ciertas propiedades de la organización, tales como:

Estructura. Está vinculada con las reglas organizacionales, los formalismos, las obligaciones, políticas, jerarquías y regulaciones. Es decir, se refiere a los canales formales dentro de la organización.

Responsabilidad. Es la percepción del individuo sobre el ser su propio jefe, el tener un compromiso elevado con el trabajo, el tomar decisiones por sí solo, el crearse sus propias exigencias.

Recompensa. Corresponde a los estímulos recibidos por el trabajo bien hecho. Es la medida en que la organización utiliza más el premio que el castigo. 
Riesgo. Corresponde al sentimiento que tienen los miembros de la organización acerca de los desafíos que impone el trabajo. Es la medida en que la organización promueve retos calculados a fin de lograr los objetivos propuestos.

Calor. Es la percepción por parte de los miembros de la empresa acerca de la existencia de un ambiente de trabajo grato y de buenas relaciones sociales tanto entre pares como entre jefes y subordinados.

Apoyo. Es el sentimiento sobre la existencia de un espíritu de ayuda de parte de los directivos y de otros empleados del grupo.

Estándares de desempeño. Es el énfasis que pone la organización sobre las normas de rendimiento. Se refiere a la importancia de percibir metas implícitas y explícitas así como normas de desempeño.

Conflicto. Es el grado en que los miembros de la organización, tanto pares como superiores, aceptan las opiniones discrepantes y no temen enfrentar y solucionar los problemas tan pronto surjan.

Identidad. Es el sentimiento de pertenencia a la organización, el cual es un elemento importante y valioso dentro del grupo de trabajo. En general, es la sensación de compartir los objetivos personales con los de la organización.

En un estudio realizado por de Frias y Schaie (2001) y Frías (2003) se encontró que existen diferencias significativas entre al percepción del clima laboral con respecto a la edad, el sexo y el tipo de ocupación. Frías menciona que los empleados con edades de entre 50 y 56 años, así como las personas que ocupan puestos gerenciales tienen una mejor percepción del clima en cuanto a autonomía, control e innovación.

Por su parte, Palma (2000) realizó un estudio de clima organizacional en una institución educativa y encontró que no existen diferencias en cuanto a la percepción del clima laboral cuando se comparan sus promedios en función al sexo, grupo ocupacional y tiempo de servicio.

El objetivo de la presente investigación es identificar el clima organizacional en una empresa cervecera, a partir de la administración del Cuestionario de clima organizacional de Litwin y Stringer (1968), así como determinar si existen diferencias entre el sexo de los trabajadores y la edad de los mismos.

\section{MÉTODO}

\section{Participantes}

Participaron en el estudio todos los empleados directos y subcontratados de la empresa HERLO. El cuestionario se administró a 49 trabajadores con un promedio de edad de 29.35 años (d.s. \pm 9.37 ). De los cuales, 32 eran hombres y 17 eran mujeres. El promedio de edad del primer grupo fue de 30.91 años (d.s. \pm 9.97 ) y del segundo de 26.41 años (d.s. \pm 7.53$)$ (Ver tabla 1). 
Tabla N. ${ }^{\circ}$ 1. Número de participantes distribuidos por Sexo y Edad promedio.

\begin{tabular}{|c|c|c|c|c|c|c|c|c|}
\hline \multicolumn{6}{|c|}{ Sexo } & \multirow{2}{*}{\multicolumn{3}{|c|}{ Total general }} \\
\hline \multicolumn{3}{|c|}{ Hombres } & \multicolumn{3}{|c|}{ Mujeres } & & & \\
\hline $\mathrm{n}$ & Media de edad & d.s. de edad & $\mathrm{n}$ & Media de edad & d.s. de edad & $\mathrm{n}$ & Media de edad & d.s. de edad \\
\hline 32 & 30.91 & 9.97 & 17 & 26.41 & 7.53 & 49 & 29.35 & 9.37 \\
\hline
\end{tabular}

\section{Descripción del instrumento}

Para medir el clima organizacional se utilizó el instrumento que fue construido por Litwin y Stringer adaptado por Echezuria y Rivas (2001) y utilizado por Marín Pérez (2003).

El cuestionario recoge y describe hechos de la organización mediante la percepción del individuo (Marín Pérez, 2003). El instrumento en su formato original está conformado por 53 reactivos distribuidos en 9 dimensiones en escala tipo Likert con cuatro opciones de respuesta. Las escalas son: estructura, responsabilidad, recompensa, riesgo, calor, apoyo, estándares de desempeño, conflicto e identidad.

\section{Conducción del estudio}

El instrumento se administró individualmente, dentro de las instalaciones administrativas de la empresa.

La participación fue voluntaria, losparticipantes no recibieron alguna compensación a cambio y en todo momento se les garantizó la confidencialidad de la información.

El tiempo en responder a los cuestionarios fue de 40 minutos en promedio.

\section{Análisis de datos}

Las calificaciones brutas obtenidas por los sujetos en el Cuestionario de clima organizacional se transformaron en calificaciones jerárquicas porcentuales. Posteriormente, se calculó el Análisis de varianza de un factor para identificar diferencias entre las escalas del cuestionario. El estadístico t de Student fue utilizado con el propósito de identificar si existen diferencias entre el sexo y la edad, cabe señalar que la edad se partió por la mediana de los valores con el fin de obtener dos grupos.

\section{RESULTADOS}

La figura N. ${ }^{\circ} 1$ presenta el promedio de las puntuaciones jerárquicas porcentuales de las escalas de clima organizacional. Como puede observarse, los empleados de la empresa cervecera tienen una buena percepción del clima en las escalas de apoyo $(\mathrm{m}=67.57$, d.s. \pm 13.39$)$, de estructura $(m=62.31$, d.s. \pm 16.10$)$, de riesgo $(m=60.14$, d.s. \pm 19.36$)$, de responsabilidad $(\mathrm{m}=58.50$, d.s.16.03) y de calor $(\mathrm{m}=56.33$, d.s. \pm 21.69$)$. No obstante, $\mathrm{su}$ percepción cambia de manera negativa en las escalas de conflicto $(m=48.30$, d.s. \pm 15.43$)$, 
identidad ( $\mathrm{m}=44.56$, d.s. \pm 20.31$)$, estándares de desempeño $(\mathrm{m}=43.67$, d.s. \pm 19.58$)$ y recompensa $(m=39.68$, d.s. \pm 21.66$)$. Al Al llevar a cabo el análisis de varianza de un factor para determinar si existen diferencias entre las medias de las escalas, los resultados reportaron diferencias estadísticamente significativas $\mathrm{F}(1,8)=13.53 ; \mathrm{p}<0.001$.

Figura N. ${ }^{0}$ 1. Promedio de puntuaciones jerárquicas porcentuales de las escalas de clima organizacional $(\mathrm{N}=49)$.

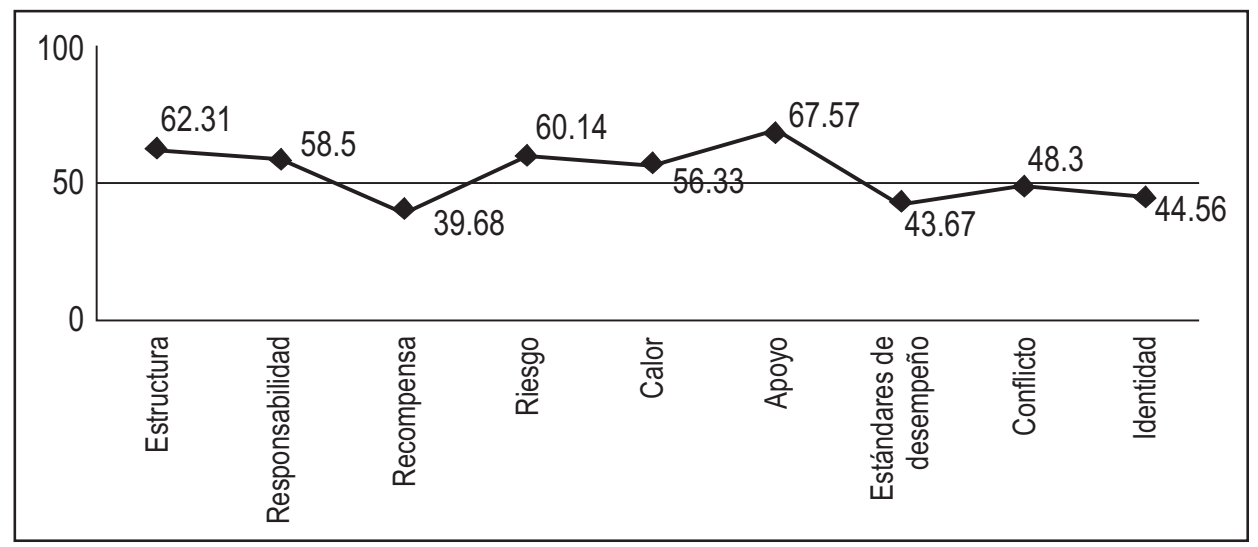

$\mathrm{F}(1,8)=13.53 ; \mathrm{p}<0.001$

Otro análisis que se realizó fue el estadístico t de Student con el propósito de identificar si existen diferencias entre hombres y mujeres con respecto a la percepción que tienen sobre el clima en su organización.

Como puede observarse en la tabla 2, los hombres obtuvieron puntuaciones más altas en las escalas de estructura, recompensa, calor, apoyo, estándares de desempeño y conflicto. Sin embargo, las mujeres puntuaron más alto en las escalas de responsabilidad, riesgo e identidad.

Se encontraron diferencias en recompensa $\mathrm{t}(2,49)=2.28 ; \mathrm{p}<0.05$ y en estándares de desempeño $\mathrm{t}(2,49)=2.64 ; \mathrm{p}<0.001$. Los hombres obtuvieron una puntuación más alta en la primera escala $(m=44.62$; d.s. \pm 22.73$)$ con respecto a las mujeres $(m=30.39$, d.s. \pm 16.33$)$ y en la misma línea, los hombres percibieron de manera más positiva el clima en la escala de estándares de desempeño $(m=48.75$, d.s. \pm 18.97$)$ que las mujeres $(\mathrm{m}=34.12$, d.s. \pm 17.46$)$. 
Tabla N. ${ }^{\circ}$ 2. Resultados del contraste de medias con respecto al sexo de los trabajadores $(\mathrm{N}=49)$.

\begin{tabular}{lccc}
\hline Dimensiones & Media Hombres & Media Mujeres & $\mathbf{t}(\mathbf{2 , 4 9 )}$ \\
\hline Estructura & 64.69 & 57.84 & 1.43 \\
Responsabilidad & 57.29 & 60.78 & 0.72 \\
Recompensa & 44.62 & 30.39 & $2.28^{*}$ \\
Riesgo & 59.58 & 61.18 & 0.27 \\
Calor & 59.58 & 50.20 & 1.46 \\
Apoyo & 68.23 & 66.34 & 0.47 \\
Estándares de desempeño & 48.75 & 34.12 & $2.64^{* *}$ \\
Conflicto & 51.04 & 43.14 & 1.74 \\
Identidad & 42.19 & 49.02 & 1.12 \\
\hline
\end{tabular}

${ }^{*} \mathrm{p}<0.05 ; * * \mathrm{p}<0.001$

Para determinar si hay diferencias en la percepción del clima con respecto a la edad de los trabajadores, se realizó el cálculo de la t de Student. Cabe resaltar que la edad se cortó por la mediana de los valores para obtener dos grupos: el primer grupo está constituido por aquellos trabajadores menores de 26 años y el segundo por los trabajadores cuyas edades oscilan de 26 o más años.

Los resultados se presentan en la tabla 3. Como puede apreciarse, los trabajadores más jóvenes obtuvieron puntuaciones más altas en ocho de las nueve escalas. No obstante, los trabajadores del segundo grupo obtuvieron una puntuación más alta en la escala de identidad $(\mathrm{m}=47.99$, d.s. \pm 17.77$)$ con respecto a los trabajadores del primer grupo $(\mathrm{m}=39.58$; d.s. \pm 23.08$)$.

Los resultados del contraste de medias no reportaron diferencias estadísticamente significativas entre los dos grupos de edades.

Tabla N. ${ }^{\circ}$ 3. Resultados contraste de medias de edad de los trabajadores $(\mathrm{N}=49)$.

\begin{tabular}{lccc}
\hline Escalas & Media $<\mathbf{2 6}$ años & Media $\geq \mathbf{2 6}$ años & $\mathbf{t}(\mathbf{2 , 4 9 )}$ \\
\hline Estructura & 65.17 & 60.34 & 1.03 \\
Responsabilidad & 59.05 & 58.13 & 0.20 \\
Recompensa & 43.06 & 37.36 & 0.90 \\
Riesgo & 63.33 & 57.93 & 0.96 \\
Calor & 58.33 & 54.94 & 0.53 \\
Apoyo & 67.78 & 67.43 & 0.09 \\
Estándares de desempeño & 45.67 & 42.30 & 0.59 \\
Conflicto & 50.67 & 46.67 & 0.89 \\
Identidad & 39.58 & 47.99 & -1.44 \\
\hline
\end{tabular}




\section{DISCUSIÓN}

El objetivo del presente trabajo fue identificar la percepción del clima organizacional en los trabajadores de una empresa cervecera. En este sentido, los resultados reportaron una buena percepción en las escalas de apoyo, estructura, riesgo, responsabilidad y calor y una mala percepción en conflicto, identidad, estándares de desempeño y recompensa.

Los resultados fueron consistentes con la realidad organizacional. Puede advertirse que aunque la organización objeto de análisis sea una empresa familiar, en donde las decisiones las toma la cúspide. La estructura organizacional no es tan rígida, lo que permite a lo empleados plena libertad para realizar el trabajo. De antemano se sabe lo que hay que hacer, cómo y cuándo (riesgo y responsabilidad).

Los empleados en general comparten el gusto por el deporte y es tradición, entre ellos, participar en torneos de fút-bol, voley-bol o realizar peregrinaciones a través del ciclismo. Esto puede repercutir positivamente, ya que se identifican unos con otros y se fomentan buenas relaciones (calor y apoyo).

La organización no cuenta con normas de desempeño y políticas de incentivos. Únicamente el sueldo de los trabajadores del área de ventas está en función de las ventas realizadas (estándares de desempeño y recompensa).

La percepción negativa en la escala de Identidad pudo deberse a la clara diferencia entre los objetivos de los empleados y los de la organización, es decir, la administración no se preocupa por fomentar la visión, la misión. Del mismo modo, ésta no hace participes a los empleados de su plan general, no comparte, ni recibe información que permita hacer crecer a la organización. En síntesis, por un lado se deja la libertad condicionada para cumplir con el deber y por el otro, se marca la diferencia entre lo que desea el empleado y el jefe. Debido a que gran parte de lo trabajadores encuestados forman parte del área administrativa, la carga de trabajo varía y existen tiempos muertos; lo que, probablemente, fomenta los rumores o se presenten conflictos.

Los resultados reportaron que los hombres tienen una mejor percepción del clima en las escalas de Estándares de Desempeño y Recompensa con respecto a las mujeres. Lo anterior puede deberse a que la mayor parte de los hombres encuestados se encuentran en el área de ventas y son los únicos en la organización que reciben su sueldo en función de las ventas realizadas. El resto de los empleados tiene un sueldo fijo.

La percepción del clima en cuanto a grupos de edades no reflejó diferencias significativas. No obstante, los trabajadores más jóvenes tienen una mejor percepción del clima en ocho de las nueve escalas. Lo anterior puede deberse a que los primeros tienen actividades fuera del ambiente laboral, lo que hace que exista cohesión entre ellos y repercuta de manera favorable en la organización.

Sin embargo, los empleados con más edad tienden a percibir un mejor clima en identidad. En este sentido, puede recalcarse que desde que se fundó la compañía, ésta era una de las pocas empresas de la región que proporcionaba a sus empleados todas las prestaciones que la Ley obliga, lo que ocasionaba que los empleados sintieran cierta seguridad en el puesto y parte de la propia organización. 


\section{CONCLUSIÓN}

El presente trabajo permitió identificar el clima de los trabajadores a partir de un enfoque integrado, es decir, la percepción de los efectos subjetivos percibidos, del sistema formal, del estilo formal de los administradores, y de otros factores ambientales importantes sobre las actitudes, creencias, valores, y motivación de las personas que trabajan en la organización (Litwin y Stringer, 1968).

En este sentido, el instrumento puede ser una herramienta de ayuda que permita a los administradores aprovechar las fortalezas manifiestas en los resultados de la investigación y realizar una intervención en los aspectos del clima relacionados con la recompensa, la identidad, los estándares de desempeño y el conflicto, las cuales son un área de mejora.

\section{REFERENCIAS BIBLIOGRÁFICAS}

1. Brunet, L. (1992) El clima de trabajo en las organizaciones: definición, diagnóstico y consecuencias. México: Trillas.

2. de Frias, C.M. y Schaie, K.W. (2001). Perceived work environment and cognitive style. Experimental Aging Research, 29, 335-344.

3. Dessler, G. (1998). Administración. México: Mc. Graw Hill.

4. DeStefano, T.J., Clark, H., Gavin, M. y Potter, T. (2005). The relationship between work environment factors and job satisfaction among rural behavioral health professionals. Journal of community psychology, 1-7.

5. Echezuria, A. y Rivas, A. (2001). Estudio de clima organizacional de la unidad de RRHH en una muestra de organismos públicos y empresas privadas. Tesis de licenciatura. Universidad Católica Andrés Bello. Caracas, Venezuela.

6. Frías Díaz, D. A. (2003). Clima organizacional entre grupos de docentes secundarios de una unidad de Servicios Educativos de Lima metropolitana. Tesis Universidad de San Martín de Porres. Lima, Perú.

7. García García, I. y Sánchez Santa-Bárbara, E. (2008). Clima psicológico y estilos de liderazgo. Revista de Trabajo y Seguridad Social. CEF, 299: 217-264.

8. Gómez Rada, C.A. (2004). Diseño, construcción y validación de un instrumento que evalúa clima organizacional en empresas colombianas, desde la teoría de la respuesta al ítem. Acta colombiana de psicología, 11, 97-113.

9. Jaime Santana, P. y Araujo Cabrera, Y. (2009). Clima y cultura organizacional: ¿Dos constructos para explicar un mismo fenómeno? Decisiones organizativas, 296-324.

10. Litwin, G.H. y Stringer, R.A. (1968). Motivation and organizational climate. Boston: Harvard Business School Press.

11. Marín Pérez, M. (2003). Relación entre clima y el compromiso organizacional en una empresa del Sector Petroquímico. Tesis de Licenciatura. Universidad Católica Andrés Bello, Facultad de Ciencias Económicas y Sociales, Caracas, Venezuela. 